\title{
A large-deviation principle for random evolution equations
}

\author{
MOHAMED MELLOUK \\ Laboratoire de Probabilités (UMR 7599), Université Paris VI, 4 place Jussieu, 75252 Paris, \\ France.E-mail:mellouk@proba.jussieu.fr
}

We consider the family of stochastic processes $X^{\varepsilon}=\left\{X^{\varepsilon}(t), 0 \leqslant t \leqslant 1\right\}, \varepsilon>0$, where $X^{\varepsilon}$ is the solution of the Itô stochastic differential equation

$$
\mathrm{d} X^{\varepsilon}(t)=\sqrt{\varepsilon} \sigma\left(X^{\varepsilon}(t), Z(t)\right) \mathrm{d} W_{t}+b\left(X^{\varepsilon}(t), Y(t)\right) \mathrm{d} t,
$$

whose coefficients depend on processes $Z(t)=\{Z(t), t \in[0,1]\}$ and $Y(t)=\{Y(t), t \in[0,1]\}$. Using an extended 'contraction principle', we give the large-deviation principle (LDP) of $X^{\varepsilon}$ as $\varepsilon \rightarrow 0$. This extends the LDP for a random evolution equation, proved by Yi-Jun Hu, to the case of random diffusion coefficients.

Keywords: Hölder spaces; large-deviation principle; random evolution equations; relative compactness

\section{Introduction}

The validity of the large-deviation principle (LDP) has important applications to various areas including statistics, especially in statistical tests (see Chernoff 1952), information theory, communication networks and risk-sensitive control. It also appears in the evaluation of the free energy in statistical mechanics (see Ruelle 1969) and Varadhan's small time estimates of the density (see Varadhan 1967). For historical background on the theory of large deviations, we refer to Deuschel and Stroock (1989) or Dembo and Zeitouni (1993).

Let $\Omega=\mathscr{C}\left([0,1], \mathbb{R}^{k}\right)$ denote the space of continuous $\mathbb{R}^{k}$-valued functions endowed with the usual topology of uniform convergence and $W$ be a standard Brownian motion on $\Omega$. Then, for $\varepsilon>0$, let $\left\{X^{\varepsilon}\right\}$ be the diffusion process solution of the stochastic differential equation (SDE)

$$
\mathrm{d} X_{t}^{\varepsilon}=b\left(X_{t}^{\varepsilon}\right) \mathrm{d} t+\sqrt{\varepsilon} \sigma\left(X_{t}^{\varepsilon}\right) \mathrm{d} W_{t}, \quad 0 \leqslant t \leqslant 1, \quad X_{0}^{\varepsilon}=x \in \mathbb{R}^{d},
$$

where $b, \sigma$ satisfy the usual Lipschitz conditions. The large-deviations theory for the law of $X^{\varepsilon}$ has been extensively studied, and it is well known that the diffusion trajectories converge exponentially fast as $\varepsilon \rightarrow 0$ to a deterministic path that minimizes the energy (see Azencott 1980; Freidlin and Wentzell 1984). This can be deduced from Schilder's theorem by means of an appropriate version of the contraction principle, known as the Freidlin-Wentzell inequality. The validity of this LDP has been recently extended to a stronger topology on $\Omega$ (see Ben Arous and Ledoux 1994).

For $0 \leqslant \alpha<\frac{1}{2}$, let $\mathscr{C}^{\alpha, 0}\left([0,1], \mathbb{R}^{d}\right)$ be the separable space of $\alpha$-Hölder continuous 
functions from $[0,1]$ to $\mathbb{R}^{d}$. The goal of this paper is to derive an LDP in $\mathscr{C}^{\alpha, 0}\left([0,1], \mathbb{R}^{d}\right)$ for the families of laws of the random evolution equations

$$
X^{\varepsilon}(t)=x+\sqrt{\varepsilon} \int_{0}^{t} \sigma\left(X^{\varepsilon}(s), Z(s)\right) \mathrm{d} W_{s}+\int_{0}^{t} b\left(X^{\varepsilon}(s), Y(s)\right) \mathrm{d} s,
$$

where $x \in \mathbb{R}^{d}, W$ is a standard Brownian motion, $Y$ is a progressively measurable random process which satisfies some integrability conditions and $Z$ is a random process such that topological support of $Z$ is a compact subset of $\mathscr{C}^{\alpha, 0}\left([0,1], \mathbb{R}^{l}\right)$. Furthermore, $W$ is independent of $(Y, Z)$ and $\sigma, b$ satisfy some regularity assumptions which we will describe later.

The special case where $Z \equiv 0$ and $\sigma \equiv I d_{d \times d}$, has been studied by Bezuidenhout (1987), who gives an explicit expression for the rate function by means of a modification of the contraction principle.

For $Z \equiv 0$ and $\sigma \not \equiv I d_{d \times d}$, such processes have been studied by many authors, among them Griego and Hersh (1971) and Heath (1969). The LDP in $\alpha$-Hölder topology, $0<\alpha<\frac{1}{2}$, is proved by $\mathrm{Hu}$ (1997). The argument in that case uses essentially a version of Theorem 4.2.16 of Dembo and Zeitouni (1993) (which is a modification of Azencott's technique).

Here, we prove the LDP for the law of the solutions of (1.1) in the case where $Z \not \equiv 0$, $\sigma \not \equiv I d_{d \times d}$, the support of $Z$ is compact and for a non-bounded drift coefficient. The problem becomes more delicate since, unlike in $\mathrm{Hu}$ (1997), the dependence of $\sigma$ upon some process $Z$ prevents $X^{\varepsilon}$ from being written as an explicit function of $\sqrt{\varepsilon} W, Y$ and $Z$. We circumvent the problem by proving another extension of the contraction principle, which generalizes that recently shown by Pérez-Abreu and Tudor (1994, Theorem 2.1), and used by these authors to prove an LDP for a class of chaos expansions.

The paper is organized as follows. Preliminary definitions and general results on the LDP are given in Section 1.1. In Section 2 the main result concerning the LDP for the solution of (1.1) is stated. Section 3 is devoted to a general approximate contraction principle that meets our needs. In Section 4 we prove that this version of the contraction principle can be applied in our framework. For a random variable $Y$, we denote by supp $Y$ the support of the distribution of $Y$.

\subsection{Definitions and general results}

Let $E$ be a topological space and $\mathscr{F}$ its Borel $\sigma$-field, and let $\left\{P_{\varepsilon} ; \varepsilon>0\right\}$ be a family of probability measures on $(E, \mathscr{F})$. We begin by giving several definitions; for more details, see Dembo and Zeitouni (1993) or Varadhan (1984).

Definition 1.1. A function $I: E \rightarrow[0, \infty]$ is said to be a rate function if it is lower semicontinuous (lsc). Furthermore, if, for each $a<\infty, \Gamma_{a}=\{x \in E ; I(x) \leqslant a\}$ is compact, we will say that I is a good rate function.

Unless explicitly stated otherwise, for any subset $A$ of $E$ and any rate function, we set

$$
I(A)=\inf _{x \in A} I(x) .
$$


Definition 1.2. For some function $I$, the probabilities $\left\{P_{\varepsilon}\right\}_{\varepsilon>0}$ satisfy a large-deviation principle if the following hold:

(i) $I$ is a good rate function.

(ii) (Lower bound.) For every open subset $G$ of $E$,

$$
\liminf _{\varepsilon \rightarrow 0} \varepsilon \ln P_{\varepsilon}(G) \geqslant-I(G) .
$$

(iii) (Upper bound.) For every closed subset F of E,

$$
\limsup _{\varepsilon \rightarrow 0} \varepsilon \ln P_{\varepsilon}(F) \leqslant-I(F)
$$

Let $\left(W=\{W(t), t \geqslant 0\}, \Omega, \mathscr{F}, \mathscr{F}_{t}, P\right)$ be $d$-dimensional standard Brownian motion and $\Omega=\mathscr{C}\left([0,1], \mathbb{R}^{d}\right)$ be the set continuous functions from $[0,1]$ to $\mathbb{R}^{d}$ equipped with the usual topology of uniform convergence defined by the norm $\|f\|_{\infty}=\sup _{0 \leqslant t \leqslant 1}|f(t)|$, and let $\mathscr{H}\left([0,1], \mathbb{R}^{d}\right)$ be the Cameron-Martin space, that is, the subset of $\Omega$ consisting of all absolutely continuous functions $h$ satisfying $h(0)=0$ and $\left.\int_{0}^{1}|\dot{h}(s)|^{2} \mathrm{~d} s\right)<\infty(\dot{h}(t)=\mathrm{d} h / \mathrm{d} t)$. For $h \in \mathscr{H}\left([0,1], \mathbb{R}^{d}\right)$ we set $\|h\|_{\mathscr{B}}=\left(\int_{0}^{1}|\dot{h}(s)|^{2} \mathrm{~d} s\right)^{1 / 2}$.

For $0<\alpha<\frac{1}{2}$, we define the $\alpha$-Hölder space $\mathscr{C}^{\alpha}\left([0,1] ; \mathbb{R}^{d}\right)$ as the space of continuous functions $f$ such that

$$
\|f\|_{\alpha}=\sup _{\{0<|t-s| \leqslant 1} \frac{|f(t)-f(s)|}{|t-s|^{\alpha}}<\infty .
$$

Define the Hölderian modulus of continuity of $f$ by

$$
\omega_{\alpha}(f, \delta)=\sup _{0<|t-s| \leqslant \delta} \frac{|f(t)-f(s)|}{|t-s|^{\alpha}} .
$$

It is well known that $\mathscr{C}^{\alpha}\left([0,1] ; \mathbb{R}^{d}\right)$ is not separable but its closed subspace, defined by

$$
\mathscr{C}^{\alpha, 0}\left([0,1] ; \mathbb{R}^{d}\right)=\left\{f \in \mathscr{C}^{\alpha}\left([0,1] ; \mathbb{R}^{d}\right) ; \lim _{\delta \rightarrow 0} \omega_{\alpha}(f, \delta)=0\right\},
$$

is separable. Both $\mathscr{C}^{\alpha}\left([0,1] ; \mathbb{R}^{d}\right)$ and $\mathscr{C}^{\alpha}\left([0,1] ; \mathbb{R}^{d}\right)$ are Banach spaces for the norm $\|\cdot\|_{\alpha}$ and $\|\cdot\|_{\infty} \leqslant\|\cdot\|_{\alpha}$. It is well known that $P\left(\|W\|_{\alpha}<\infty\right)=1$ for $0<\alpha<\frac{1}{2}$. We shall need the following version of the Arzelà-Ascoli theorem.

Theorem 1.3. $A$ set $A \subset \mathscr{C}^{\alpha, 0}\left([0,1] ; \mathbb{R}^{d}\right)$ has compact closure in $\mathscr{C}^{\alpha, 0}\left([0,1] ; \mathbb{R}^{d}\right)$ if and only if the following two conditions hold:

$$
\sup _{f \in A}\|f\|_{\alpha}<\infty
$$

and

$$
\lim _{\delta \downarrow 0} \sup _{f \in A} \omega_{\alpha}(f, \delta)=0 .
$$

The following LDP proved by Baldi et al. (1992) extends the classical Schilder theorem (see Schilder 1966; Deuschel and Stroock 1989). 
Theorem 1.4. The probability measures induced by $\sqrt{\varepsilon} W$ on $\mathscr{C}^{\alpha, 0}\left([0,1] ; \mathbb{R}^{d}\right)$, equipped with the norm $\|\cdot\|_{\alpha}$, satisfy the LDP with the good rate function $\lambda(\cdot)$ defined by

$$
\lambda(h)= \begin{cases}\frac{1}{2} \int_{0}^{1}|\dot{h}(s)|^{2} \mathrm{~d} s, & \text { if } h \in \mathscr{H}\left([0,1], \mathbb{R}^{d}\right), \\ +\infty, & \text { otherwise }\end{cases}
$$

We now state the Hölder version of classical exponential inequality for stochastic integrals, which is crucial in proving the exponential approximation (see, for example, Stroock 1981, Lemma 8.27).

Lemma 1.5. Let $f:[0,1] \times \Omega \rightarrow \mathbb{R}^{\prime} \otimes \mathbb{R}^{d}$ and $g:[0,1] \times \Omega \rightarrow \mathbb{R}^{\prime}$ be bounded $\left\{\mathscr{F}_{t}\right\}$ progressively measurable functions, and set

$$
U(t)=\int_{0}^{t} f(s) \mathrm{d} W_{s}+\int_{0}^{t} g(s) \mathrm{d} s, \quad 0 \leqslant t \leqslant 1 .
$$

Define $A=\sup _{t, \omega} \operatorname{tr}\left(f(t, \omega) f^{\mathrm{T}}(t, \omega)\right)$ and $B=\sup _{t, \omega}|g(t, \omega)|$. Then, for every $s \geqslant 0, T \geqslant 0$, $0 \leqslant \alpha<\frac{1}{2}$ and $r>\ell B T^{1-\alpha}$,

$$
P\left(\sup _{s \leqslant t \leqslant s+T} \frac{|U(t)-U(s)|}{|t-s|^{\alpha}} \geqslant r\right) \leqslant 2 \ell \exp \left[-\frac{\left(r-\ell B T^{1-\alpha}\right)^{2}}{2 A \ell^{2} T^{1-2 \alpha}}\right] .
$$

\section{The main result}

In this section we give conditions under which the solution of the SDE defined by (1.1) satisfies an LDP in any Hölder norm with exponent $\alpha<\frac{1}{2}$.

Let $\Omega=\mathscr{C}\left([0,1], \mathbb{R}^{k}\right)$ be the space of trajectories of a standard $\mathbb{R}^{k}$-valued Brownian motion $\left(W_{t}, 0 \leqslant t \leqslant 1\right), P$ the Wiener measure and $\mathscr{T}$ the completion of the Borel $\sigma$-field of $\Omega$ with respect to $P$. Let $Y=\{Y(t) ; 0 \leqslant t \leqslant 1\}$ be a $\mathbb{R}^{m}$-valued process which is $\left\{\mathscr{F}_{t}\right\}$ progressively measurable. In order to make explicit the LDP rate function for the law of (1.1) in the $\alpha$-Hölder topology, we suppose that $Y$ is a random variable with values in $L^{1 /(1-\alpha)}\left([0,1], \mathbb{R}^{m}\right)$. Let $Z=\{Z(t) ; 0 \leqslant t \leqslant 1\}$ be an $\left\{\mathscr{F}_{t}\right\}$-progressively measurable process taking values in $\mathbb{R}^{l}$. We assume that $\operatorname{supp} Z$ is a compact subset in $\mathscr{C}^{\alpha, 0}\left([0,1], \mathbb{R}^{l}\right)$, and that $(Y, Z)$ and $W$ are independent.

From now on, we suppose that the coefficients $\sigma(\cdot, \cdot)$ and $b(\cdot, \cdot)$ satisfy the following hypotheses:

$\left(\mathrm{H}_{0}\right) \sigma: \mathbb{R}^{d} \times \mathbb{R}^{l} \rightarrow \mathbb{R}^{d} \otimes \mathbb{R}^{k}$ and $b: \mathbb{R}^{d} \times \mathbb{R}^{m} \rightarrow \mathbb{R}^{d}$.

$\left(\mathrm{H}_{1}\right)$ The function $b(x, y)$ is jointly measurable in $(x, y)$ and there exists a constant

$C>0$ such that 


$$
\begin{aligned}
|b(x, y)| & \leqslant C(1+|x|), \quad \forall(x, y) \in \mathbb{R}^{d} \times \mathbb{R}^{m}, \\
\left|b(x, y)-b\left(x^{\prime}, y^{\prime}\right)\right| & \leqslant C\left(\left|x-x^{\prime}\right|+\left|y-y^{\prime}\right|\right), \quad \forall x, x^{\prime} \in \mathbb{R}^{d}, \quad \forall y, y^{\prime} \in \mathbb{R}^{m} .
\end{aligned}
$$

$\left(\mathrm{H}_{2}\right)$ The function $\sigma(x, z)$ is jointly measurable in $(x, z)$ and there exists a constant $C>0$ such that

$$
\begin{aligned}
\|\sigma(x, z)\| & \leqslant C, \quad \forall(x, z) \in \mathbb{R}^{d} \times \mathbb{R}^{l}, \\
\left\|\sigma(x, z)-\sigma\left(x^{\prime}, z^{\prime}\right)\right\| & \leqslant C\left(\left|x-x^{\prime}\right|+\left|z-z^{\prime}\right|\right), \quad \forall x, x^{\prime} \in \mathbb{R}^{d}, \quad \forall z, z^{\prime} \in \mathbb{R}^{l} .
\end{aligned}
$$

For $\varepsilon>0$, let $\left\{X^{\varepsilon}(t) ; 0 \leqslant t \leqslant 1\right\}$ be the process satisfying the SDE

$$
X^{\varepsilon}(t)=x+\sqrt{\varepsilon} \int_{0}^{t} \sigma\left(X^{\varepsilon}(s), Z(s)\right) \mathrm{d} W_{s}+\int_{0}^{t} b\left(X^{\varepsilon}(s), Y(s)\right) \mathrm{d} s, \quad x \in \mathbb{R}^{d} .
$$

The existence of a unique solution of (2.1), which is $\left\{\mathscr{F}_{t}\right\}$-adapted and has $\alpha$-Hölder continuous sample paths, is ensured by $\left(\mathrm{H}_{0}\right)-\left(\mathrm{H}_{2}\right)$ and standard results on existence and uniqueness of solutions of SDEs with random coefficients (see, for example, Gihman and Skorohod 1972, Section 5.5, Theorem 1).

For $h \in \mathscr{H}\left([0,1], \mathbb{R}^{d}\right), r \in L^{1 /(1-\alpha)}\left([0,1], \in \mathbb{R}^{m}\right)$ and $u \in \operatorname{supp} Z$, let $\phi(h, r, u)(\cdot)$ denote the unique solution of the ordinary differential equation

$$
g(t)=x+\int_{0}^{t} \sigma(g(s), u(s)) \dot{h}(s) \mathrm{d} s+\int_{0}^{t} b(g(s), r(s)) \mathrm{d} s, \quad x \in \mathbb{R}^{d}, \quad t \in[0,1] .
$$

The existence and uniqueness of the solution (2.2) is a consequence of the Lipschitz continuity of $\sigma$ and $b$ and is standard. Define $\tilde{\lambda}: \mathscr{C}^{\alpha, 0}\left([0,1], \mathbb{R}^{d}\right) \rightarrow[0, \infty]$ by

$$
\tilde{\lambda}(\tilde{h})=\inf \left\{\lambda(h) ; h \in \mathscr{H}\left([0,1], \mathbb{R}^{d}\right): \exists(r, u) \in \operatorname{supp} Y \times \operatorname{supp} Z \text { s.t. } \phi(h, r, u) \equiv \tilde{h}\right\} .
$$

Since $\tilde{\lambda}$ is not necessarily lsc (see, for example, Bezuidenhout 1987, p. 651), we introduce its lsc regularization $\tilde{\lambda}^{*}$ defined by

$$
\tilde{\lambda}^{*}(\tilde{h})=\lim _{a \rightarrow 0} \inf _{\rho \in B_{a}(\tilde{h}, a)} \tilde{\lambda}(\rho),
$$

where $B_{\alpha}(\tilde{\rho}, a)$ is the ball of radius $a$ centred at $\tilde{\rho}$ with respect the norm $\|\cdot\|_{\alpha}$. The existence of the limit on the right-hand side of (2.4) is ensured by the fact that $\inf _{\rho \in B_{\alpha}(\tilde{h}, a)} \tilde{\lambda}(\rho)$ is a decreasing function of $a$.

The main result of the paper is the following:

Theorem 2.1. Suppose $\left(\mathrm{H}_{0}\right)-\left(\mathrm{H}_{2}\right)$ hold. Then

(i) $\tilde{\lambda}^{*}$ defined by (2.4) is a good rate function (with respect the topology of $\left.\mathscr{C}^{\alpha, 0}\left([0,1] ; \mathbb{R}^{d}\right), 0 \leqslant \alpha<\frac{1}{2}\right)$;

(ii) The family $P^{\varepsilon}=P \circ\left(X^{\varepsilon}\right)^{-1}$ of the laws of $X^{\varepsilon}$ defined by (2.1) satisfies on $\mathscr{C}^{a, 0}\left([0,1] ; \mathbb{R}^{d}\right)$ an LDP with good rate function $\tilde{\lambda}^{*}$. 
Remark 2.2. It is natural to ask what happens if $Y$ and $Z$ also depend on $\varepsilon$. This is addressed by Bezuidenhout (1987, Proposition 2.17) and Dembo and Zeitouni (1993, Exercise 5.6.27).

\section{An extension of the contraction principle}

One of the basic tools in large-deviation theory is the 'contraction principle' (see Deuschel and Stroock 1989). It enables the new rate function to be computed after the data have been transformed by a continuous map. Let $\left\{P_{\varepsilon}\right\}$ be a family of probability measures on a space $E$ satisfying an LDP with good rate function $I$, and let $F: E \rightarrow E^{\prime}$ be continuous. Let $Q_{\varepsilon} \equiv P_{\varepsilon} \circ F^{-1}$ denote the family of image measures. Then we have the following:

Theorem 3.1. $\left\{Q_{\varepsilon}\right\}$ obeys an LDP with good rate function $J$, defined by

$$
J(y)=\inf _{\{x: F(x)=y\}} I(x),
$$

with the usual convention that inf $\varnothing=+\infty$.

Dawson and Gärtner (1987), Deuschel and Stroock (1989), Dembo and Zeitouni (1993) and recently Pérez-Abreu and Tudor (1994) have presented various extensions of this theorem to non-continuous functions.

This section is devoted to proving another contraction principle well suited for our situation.

Let $\left(E_{X}, d_{X}\right),\left(E_{Y}, d_{Y}\right),\left(E_{Z}, d_{Z}\right),\left(E^{\prime}, d^{\prime}\right)$ denote Polish spaces and $(\Omega, \mathscr{F}, P)$ be a probability space. Suppose that $\left\{X^{\varepsilon} ; \varepsilon>0\right\}$ is a family of random variables with values in $E_{X}, Y$ is a random variable with values in $E_{Y}$, and $Z$ is a random variable with values in $E_{Z}$. Given a rate function $I$ on $E_{X}$ and $a>0$, set $\Gamma_{a}=\left\{x \in E_{X}: I(x) \leqslant a\right\}$ and $\Gamma_{\infty}=\cup_{a} \Gamma_{a}$.

Theorem 3.2. Let $I$ be a good rate function on $E_{X}, F_{N}, F: \Gamma_{\infty} \times E_{Y} \times E_{Z} \rightarrow E^{\prime}$, $X_{N}^{\varepsilon}, X^{\varepsilon}: \Omega \rightarrow E^{\prime}$, be applications such that the following hold:

(a)

(i) For all $a>0$ and $N \geqslant 1,\left.F_{N}\right|_{\Gamma_{a} \times \operatorname{supp} Y \times \operatorname{supp} Z}$ is continuous.

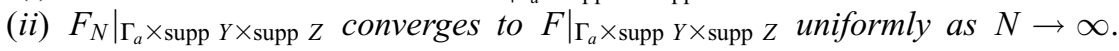

(b) For each $a>0$ and $N \geqslant 1, F_{N}(\{I \leqslant a\} \times \operatorname{supp} Y \times \operatorname{supp} Z)$ and $F(\{I \leqslant a\} \times$ supp $Y \times \operatorname{supp} Z)$ are relatively compact in $\left(E^{\prime}, d^{\prime}\right)$.

(c) For all $N \geqslant 1,\left\{X_{N}^{\varepsilon} ; \varepsilon>0\right\}$ satisfies an LDP (as $\varepsilon \rightarrow 0$ ) on $E^{\prime}$ with good rate function

$$
I_{N}^{*}(\zeta)=\lim _{\rho \rightarrow 0} \inf _{\xi \in B^{\prime}(\xi, \rho)} I_{N}(\xi),
$$

where $B^{\prime}(\xi, \rho)$ denotes the ball of radius $\rho$ centred at $\zeta$ in $\left(E^{\prime}, d^{\prime}\right)$, and

$$
I_{N}(\xi)=\inf \left\{I(x) ; \exists(y, z) \in \operatorname{supp} Y \times \operatorname{supp} Z \text { such that } F_{N}(x, y, z)=\xi\right\} .
$$


(d) $\left\{X_{N}^{\varepsilon}\right\}$ are exponentially good approximations of $\left\{X^{\varepsilon}\right\}$; that is for every $\delta>0$,

$$
\lim _{N} \limsup _{\varepsilon \rightarrow 0} \varepsilon \ln P\left\{d^{\prime}\left(X_{N}^{\varepsilon}, X^{\varepsilon}\right)>\delta\right\}=-\infty .
$$

Then $\left\{X^{\varepsilon} ; \varepsilon>0\right\}$ satisfies an LDP on $E^{\prime}$ with good rate function

$$
\tilde{I}^{*}(\zeta)=\lim _{\rho \rightarrow 0} \inf _{\xi \in B^{\prime}(\zeta, \rho)} \tilde{I}(\xi),
$$

where

$$
\tilde{I}(\xi)=\inf \{I(x) ; \exists(y, z) \in \operatorname{supp} Y \times \operatorname{supp} Z \text { such that } F(x, y, z)=\xi\} .
$$

Proof. Since $\tilde{I}^{*}$ is lsc by construction, to prove that it is a good rate function it suffices to prove that $\{\tilde{I} \leqslant a\}$ is relatively compact in $E^{\prime}$ for each $a<\infty$ (see, for example, Bezuidenhout 1987, Note 4.2). Note that

$$
\{\tilde{I} \leqslant a\} \subset\{F(x, y, z) ; x \in\{I \leqslant a+1\}, y \in \operatorname{supp} Y, z \in \operatorname{supp} Z\} .
$$

Indeed, $\tilde{I}(\xi) \leqslant a$ implies that, for all $\delta>0$, there exists $(x, y, z) \in \Gamma_{\infty} \times \operatorname{supp} Y \times \operatorname{supp} Z$ such that $F(x, y, z)=\xi$ and $I(x)<\tilde{I}(\xi)+\delta$, so that $I(x)<\tilde{I}(\xi)+\delta \leqslant a+\delta$. Using (3.2), it is enough to prove that

$$
\mathscr{K}=\{F(x, y, z) ; x \in\{I \leqslant a\}, y \in \operatorname{supp} Y, z \in \operatorname{supp} Z\}
$$

is relatively compact in $E^{\prime}$, which holds by assumption $(b)$. The same argument shows that $I_{N}^{*}$ is also a good rate function.

To prove the large-deviations lower bound, let $G$ be an open subset of $E^{\prime}$, such that $\tilde{I}^{*}(G)<\infty$; it suffices to prove that

$$
\liminf _{\varepsilon \rightarrow 0} \varepsilon \ln P\left(X^{\varepsilon} \in G\right) \geqslant-\tilde{I}(G) .
$$

Indeed, if (3.3) is true for each non-empty open set $G$, then for any $\zeta \in G$, there exists $\eta>0$ such that $B(\zeta, \eta) \subset G$; applying (3.3) with $B(\zeta, \eta)$ instead of $G$, we obtain

$$
\begin{aligned}
\liminf _{\varepsilon \rightarrow 0} \varepsilon \ln P\left(X^{\varepsilon} \in G\right) & \geqslant \liminf _{\varepsilon \rightarrow 0} \varepsilon \ln P\left(X^{\varepsilon} \in B(\zeta, \eta)\right) \\
& \geqslant-\inf \{\tilde{I}(\xi), \xi \in B(\zeta, \eta)\} .
\end{aligned}
$$

Now let $\eta \rightarrow 0$; then

$$
\liminf _{\varepsilon \rightarrow 0} \varepsilon \ln P\left(X^{\varepsilon} \in G\right) \geqslant-\tilde{I}^{*}(\zeta) \quad \text { for each } \zeta \in G,
$$

and therefore

$$
\liminf _{\varepsilon \rightarrow 0} \varepsilon \ln P\left(X^{\varepsilon} \in G\right) \geqslant-\inf \left\{\tilde{I}^{*}(\zeta) ; \zeta \in G\right\} .
$$

For any open set $\mathscr{O}$, define

$$
\begin{gathered}
J_{N}(\mathscr{O})=\inf \left\{I(x) ; x \in \Gamma_{\infty}, \exists(y, z) \in \operatorname{supp} Y \times \operatorname{supp} Z \text { s.t. } F_{N}(x, y, z) \in \mathscr{O}\right\}, \\
J(\mathscr{O})=\inf \left\{I(x) ; h \in \Gamma_{\infty}, \exists(y, z) \in \operatorname{supp} Y \times \operatorname{supp} Z \text { s.t. } F(x, y, z) \in \mathscr{O}\right\} ;
\end{gathered}
$$


then

$$
\limsup _{N} J_{N}(\mathscr{O}) \leqslant J(\mathscr{O}) .
$$

Indeed, if $J(\mathscr{O})=\infty$ we have nothing to prove; if $J(\mathscr{O})<\infty$, then, for all $\eta>0$, there exists $\left(x_{0}, y_{0}, z_{0}\right) \in \Gamma_{\infty} \times \operatorname{supp} Y \times \operatorname{supp} Z$ such that $F\left(x_{0}, y_{0}, z_{0}\right) \in \mathscr{O}$ and $J \leqslant I\left(x_{0}\right)<J(\mathscr{O})+\eta$. Choose $N_{0}>0$ such that $F_{N}\left(x_{0}, y_{0}, z_{0}\right) \in \mathscr{O}$ for $N>N_{0}$. Hence $J_{N}(\mathscr{O}) \leqslant I\left(x_{0}\right)$, for $N>N_{0}$, and consequently $\lim \sup _{N} J_{N}(\mathscr{O}) \leqslant I\left(x_{0}\right)$. Letting $\delta \rightarrow 0$, we obtain (3.4).

We now check (3.3). As usual, we have to prove that if $\xi \in G$ and $\tilde{I}(\xi)<\infty$, then

$$
\liminf _{\varepsilon \rightarrow 0} \varepsilon \ln P\left(X^{\varepsilon} \in G\right) \geqslant-\tilde{I}(\xi) .
$$

Choose $\eta>0$ such that $B(\xi, 2 \eta) \subset G$. Note that, for every $N \geqslant 1$ and $\varepsilon>0$,

$$
\left\{X_{N}^{\varepsilon} \in B(\xi, \eta)\right\} \subset\left\{X^{\varepsilon} \in G\right\} \cup\left\{d^{\prime}\left(X_{N}^{\varepsilon}, X^{\varepsilon}\right) \geqslant \eta\right\} .
$$

Hence, the large-deviation lower bounds for $\left\{X_{N}^{\varepsilon}\right\}$, and the fact that $I_{N} \geqslant I_{N}^{*}$, imply

$$
\begin{aligned}
-\inf \left\{I_{N}(\zeta) ; \zeta \in B(\xi, \eta)\right\} & \leqslant \liminf _{\varepsilon \rightarrow 0} \varepsilon \ln P\left(X_{N}^{\varepsilon} \in B(\xi, \eta)\right) \\
& \leqslant \liminf _{\varepsilon \rightarrow 0} \varepsilon \ln \left[P\left(X^{\varepsilon} \in G\right)+P\left(d^{\prime}\left(X_{N}^{\varepsilon}, X^{\varepsilon}\right) \geqslant \eta\right)\right] \\
& \leqslant \max \left\{\liminf _{\varepsilon \rightarrow 0} \varepsilon \ln P\left(X^{\varepsilon} \in G\right), \limsup _{\varepsilon \rightarrow 0} \varepsilon \ln P\left(d^{\prime}\left(X_{N}^{\varepsilon}, X^{\varepsilon}\right) \geqslant \eta\right)\right\} .
\end{aligned}
$$

Because $\left\{X_{N}^{\varepsilon}\right\}$ are exponentially good approximations of $\left\{X^{\varepsilon}\right\}$ (hypothesis $(d)$ ),

$$
\liminf _{\varepsilon \rightarrow 0} \varepsilon \ln P\left(X^{\varepsilon} \in G\right) \geqslant \liminf _{N}\left\{-\inf \left\{I_{N}(\zeta) ; \zeta \in B^{\prime}(\xi, \eta)\right\}\right\} .
$$

By (3.1) and (3.4), we have

$$
\begin{aligned}
\liminf _{N}\left\{-\inf \left\{I_{N}(\zeta) ; \zeta \in B(\xi, \eta)\right\}\right\} & =-\limsup _{N \rightarrow \infty} J_{N}\left(B^{\prime}(\xi, \eta)\right) \\
& \geqslant-J\left(B^{\prime}(\xi, \eta)\right) \geqslant-\tilde{I}(\xi)
\end{aligned}
$$

this completes the proof of the large-deviation lower bound.

In order to prove the upper bound, we first show, as in Deuschel and Stroock (1989, p. 38), that, for

$$
\ell=\lim _{\delta \rightarrow 0} \liminf _{N \rightarrow \infty}\left[\inf \left\{I(x) ; \exists(y, z) \in \operatorname{supp} Y \times \operatorname{supp} Z: d^{\prime}\left(F_{N}(x, y, z), A\right) \leqslant \delta\right\}\right],
$$

we have

$$
\inf _{\xi \in A} \tilde{I}^{*}(\xi) \leqslant \ell .
$$

To this end, suppose that $\ell<\infty$ and choose $\left(x_{p}, y_{p}, z_{p}\right) \in \Gamma_{\ell+1} \times \operatorname{supp} Y \times \operatorname{supp} Z$ and $N(p) \rightarrow \infty$ so that, for each $p \in \mathbb{N}$,

$$
d^{\prime}\left(F_{N(p)}\left(x_{p}, y_{p}, z_{p}\right), A\right) \leqslant \frac{1}{p}, \quad I\left(x_{p}\right) \leqslant \ell+\frac{1}{p} .
$$


Furthermore, if we set $g_{p}=F\left(x_{p}, y_{p}, z_{p}\right)$, then hypothesis $(a)$ ensures that, for $\rho>0$, there exists $p_{0}>0$ such that $p \geqslant p_{0}$ implies

$$
d^{\prime}\left(g_{p}, A\right) \leqslant \frac{1}{p}+\rho
$$

Since $F\left(\Gamma_{\ell+1} \times \operatorname{supp} Y \times \operatorname{supp} Z\right)$ is relatively compact in $E^{\prime}$ (hypothesis $(b)$ ), we may and we do assume that $x_{p} \rightarrow x^{*} \in \Gamma_{\ell}$ and $g_{p} \rightarrow g$ in $E^{\prime}$, as $p \rightarrow \infty$.

Clearly $g \in \bar{A}=A$, and since $\tilde{I}^{*}$ is lsc,

$$
\tilde{I}^{*}(A) \leqslant \tilde{I}^{*}(g) \leqslant \liminf _{p} \tilde{I}^{*}\left(g_{p}\right) \leqslant \liminf _{p} \tilde{I}\left(g_{p}\right) \leqslant \liminf _{p} I\left(x_{p}\right) \leqslant \ell .
$$

Observe that, for $N \geqslant 1$ and $\varepsilon>0$,

$$
\left\{X^{\varepsilon} \in A\right\} \subset\left\{X_{N}^{\varepsilon} \in \bar{A}^{\delta}\right\} \cup\left\{d^{\prime}\left(X_{N}^{\varepsilon}, X^{\varepsilon}\right) \geqslant \delta\right\},
$$

where $\bar{A}^{\delta}=\left\{\zeta: d^{\prime}(\xi, A) \leqslant \delta\right\}$ is the closed $\delta$-neighbourhood of $A$. Thus, the large-deviation upper bound for $\left\{X_{N}^{\varepsilon}\right\}$ (as $\varepsilon \rightarrow 0$ ) implies that, for every $N \geqslant 1$,

$$
\begin{aligned}
\limsup _{\varepsilon \rightarrow 0} \varepsilon \ln P\left(X^{\varepsilon} \in A\right) & \leqslant \max \left\{\limsup _{\varepsilon \rightarrow 0} \varepsilon \ln P\left(X_{N}^{\varepsilon} \in \bar{A}^{\delta}\right), \limsup _{\varepsilon \rightarrow 0} \varepsilon \ln P\left(d^{\prime}\left(X_{N}^{\varepsilon}, X^{\varepsilon}\right) \geqslant \delta\right)\right\} \\
& \leqslant \max \left\{\left[-\inf _{\xi \in \bar{A}^{\delta}} I_{N}(\xi)\right], \limsup _{\varepsilon \rightarrow 0} \varepsilon \ln P\left(d^{\prime}\left(X_{N}^{\varepsilon}, X^{\varepsilon}\right) \geqslant \delta\right)\right\} .
\end{aligned}
$$

Hence, as $\left\{X_{N}^{\varepsilon}\right\}$ are exponentially good approximations of $\left\{X^{\varepsilon}\right\}$ (assumption $(d)$ ), letting $N \rightarrow \infty$, we obtain, for every $\delta>0$,

$$
\begin{aligned}
& \limsup _{\varepsilon \rightarrow 0} \varepsilon \ln P\left(X^{\varepsilon} \in A\right) \\
& \quad \leqslant \limsup _{N}\left\{-\inf _{\xi \in \bar{A}^{\delta}} I_{N}(\xi)\right\} \\
& \quad=-\liminf _{N}\left[\inf \left\{I(x) ; \exists(y, z) \in \operatorname{supp} Y \times \operatorname{supp} Z: d^{\prime}\left(F_{N}(x, y, z), A\right) \leqslant \delta\right\}\right] .
\end{aligned}
$$

Letting $\delta \rightarrow 0$ in (3.8) and using (3.7), we obtain

$$
\limsup _{\varepsilon \rightarrow 0} \varepsilon \ln P\left(X^{\varepsilon} \in A\right) \leqslant-\inf \{\tilde{I}(\xi) ; \xi \in A\}
$$

this completes the proof, since $\tilde{I}^{\star} \leqslant \tilde{I}$.

\section{Proof of Theorem 2.1}

This section is devoted to proving Theorem 2.1 by means of Theorem 3.2. Throughout the section, $Y=\{Y(t), 0 \leqslant t \leqslant 1\}$ and $Z=\{Z(t), 0 \leqslant t \leqslant 1\}$ are the processes defined in Section 2.

In order to apply Theorem 3.2, we use the following notation: for $0 \leqslant \alpha<\frac{1}{2}$, set 


$$
\begin{aligned}
& \left(E_{X}, d_{X}\right)=\left(\mathscr{C}^{\alpha, 0}\left([0,1] ; \mathbb{R}^{k}\right),\|\cdot\|_{\alpha}\right), \\
& \left(E_{Y}, d_{Y}\right)=\left(L^{1 /(1-\alpha)}\left([0,1], \mathbb{R}^{m}\right),\|\cdot\|_{1 / L(1-\alpha)}\right), \\
& \left(E_{Z}, d_{Z}\right)=\left(\mathscr{C}^{\alpha, 0}\left([0,1] ; \mathbb{R}^{l}\right),\|\cdot\|_{\alpha}\right), \\
& \left(E^{\prime}, d^{\prime}\right)=\left(\mathscr{C}^{\alpha, 0}\left([0,1] ; \mathbb{R}^{d}\right),\|\cdot\|_{\alpha}\right)
\end{aligned}
$$

and the rate function $I$ is equal to $\lambda$ defined by (1.3). In the following, for $a<\infty$, set $\Gamma_{a}=\{\lambda \leqslant a\}, \Gamma_{\infty}=\cup_{a} \Gamma_{a}$.

For $\varepsilon>0, \quad N \geqslant 1$, set $\underline{t}_{N}=[N t] / N \quad([y]$ is the integer part of $y)$. Let $X^{\varepsilon}=$ $\left\{X^{\varepsilon}(t), \leqslant t \leqslant 1\right\}$ be the solution of $(2.1)$ and $X_{N}^{\varepsilon}=\left\{X_{N}^{\varepsilon}(t), \leqslant t \leqslant 1\right\}$ be the solution of the SDE

$$
\mathrm{d} X_{N}^{\varepsilon}(t)=\sqrt{\varepsilon} \sigma\left(X_{N}^{\varepsilon}\left(\underline{t}_{N}\right), Z\left(\underline{t}_{N}\right)\right) \mathrm{d} W_{t}+b\left(X_{N}^{\varepsilon}(t), Y(t)\right) \mathrm{d} t, \quad X_{N}^{\varepsilon}(0)=x .
$$

For $(r, u) \in L^{1 /(1-\alpha)}\left([0,1], \mathbb{R}^{m}\right) \times \mathscr{C}^{\alpha, 0}\left([0,1], \mathbb{R}^{l}\right)$ and $t \in[0,1]$, if $h \in \mathscr{C}^{\alpha, 0}\left([0,1] ; \mathbb{R}^{d}\right)$ then set

$$
\begin{aligned}
F_{N}(h, r, u)(t)= & x+\sum_{k=1}^{N} \sigma\left(F_{N}(h, r, u)\left(\frac{k-1}{N}\right), u\left(\frac{k-1}{N}\right)\right)\left\{h\left(\frac{k}{N} \wedge t\right)-h\left(\frac{k-1}{N} \wedge t\right)\right\} \\
& +\int_{0}^{t} b\left(F_{N}(h, r, u)(s), r(s)\right) \mathrm{d} s
\end{aligned}
$$

and if $h \in \mathscr{H}\left([0,1], \mathbb{R}^{d}\right)$ then set

$$
F(h, r, u)(t)=x+\int_{0}^{t} \sigma(F(h, r, u)(s), u(s)) \dot{h}(s) \mathrm{d} s+\int_{0}^{t} b(F(h, r, u)(s), r(s)) \mathrm{d} s .
$$

The existence and uniqueness of the solution of (4.1) follows from hypotheses $\left(\mathrm{H}_{0}\right)-\left(\mathrm{H}_{2}\right)$ on the coefficients and the theory of ordinary differential equations. Furthermore, the trajectories of $X^{\varepsilon}$ and $X_{N}^{\varepsilon}$ belong almost surely to $\mathscr{C}^{\alpha, 0}\left([0,1] ; \mathbb{R}^{d}\right)$.

Clearly, the process $X_{N}^{\varepsilon}$ defined by (4.1) satisfies

$$
X_{N}^{\varepsilon} \equiv F_{N}(\sqrt{\varepsilon} W, Y, Z) \text {. }
$$

In what follows, to prove Theorem 2.1, we will follow step by step the assumptions of Theorem 3.2.

\subsection{Continuity of $F_{N}$}

We prove that $F_{N}$ is continuous from $\mathscr{C}^{\alpha, 0}\left([0,1], \mathbb{R}^{d}\right) \times L^{1 /(1-\alpha)}\left([0,1], \mathbb{R}^{m}\right) \times$ $\mathscr{C}^{\alpha, 0}\left([0,1], \mathbb{R}^{l}\right)$ to $\mathscr{C}^{\alpha, 0}\left([0,1], \mathbb{R}^{d}\right)$. Fix $N \geqslant 1$ and let

$$
\left(h_{1}, r_{1}, u_{1}\right),\left(h_{2}, r_{2}, u_{2}\right) \in \mathscr{C}^{\alpha, 0}\left([0,1], \mathbb{R}^{d}\right) \times L^{1 /(1-\alpha)}\left([0,1], \mathbb{R}^{m}\right) \times \mathscr{C}^{\alpha, 0}\left([0,1], \mathbb{R}^{l}\right) ;
$$

then set

$$
F_{N}^{(i)}(\cdot)=F_{N}\left(h_{i}, r_{i}, u_{i}\right)(\cdot), \quad i=1,2
$$


and

$$
\Psi_{N}(\cdot)=F_{N}^{(1)}(\cdot)-F_{N}^{(2)}(\cdot)
$$

Lemma 4.1. Given $C>0$, we prove the existence of a constant $C_{N}>0$ (depending on $N$ and C) such that, for $\left\|h_{1}\right\|_{\alpha} \vee\left\|h_{2}\right\|_{\alpha} \leqslant C$,

$$
\left\|\Psi_{N}(\cdot)\right\|_{\alpha} \leqslant C_{N}\left\{\left\|h_{1}-h_{2}\right\|_{\alpha}+\left\|r_{1}-r_{2}\right\|_{1 / L(1-\alpha)}+\left\|u_{1}-u_{2}\right\|_{\infty}\right\} .
$$

Proof. Clearly, we have

$$
\left\|\Psi_{N}(\cdot)\right\|_{\alpha} \leqslant 2 \max \left(N^{\alpha} \sup _{t \in[0,1]}\left|\Psi_{N}(t)\right|, \max _{1 \leqslant k \leqslant N}\left\{\sup _{\frac{k-1}{N} \leqslant s<t \leqslant \frac{k}{N}} \frac{\left|\Psi_{N}(t)-\Psi_{N}(s)\right|}{|t-s|^{\alpha}}\right\}\right) .
$$

We at first show that, for $\left\|h_{1}\right\|_{\infty} \vee\left\|h_{2}\right\|_{\infty} \leqslant C$, there exists $C_{N}>0$, such that

$$
\left\|\Psi_{N}(\cdot)\right\|_{\infty} \leqslant C_{N}\left\{\left\|h_{1}-h_{2}\right\|_{\infty}+\left\|r_{1}-r_{2}\right\|_{1 / L(1-\alpha)}+\left\|u_{1}-u_{2}\right\|_{\infty}\right\} .
$$

For $t \in[0,1]$, we have

$$
\begin{aligned}
\Psi_{N}(t)= & \sum_{k=1}^{N}\left\{\sigma\left(F_{N}^{(1)}\left(\frac{k-1}{N}\right), u_{1}\left(\frac{k-1}{N}\right)\right)-\sigma\left(F_{N}^{(2)}\left(\frac{k-1}{N}\right), u_{2}\left(\frac{k-1}{N}\right)\right)\right\} \\
& \times\left\{h_{2}\left(\frac{k}{N} \wedge t\right)-h_{2}\left(\frac{k-1}{N} \wedge t\right)\right\}+\sum_{k=1}^{N} \sigma\left(F_{N}^{(1)}\left(\frac{k-1}{N}\right), u_{1}\left(\frac{k-1}{N}\right)\right) \\
& \times\left\{\left[h_{1}\left(\frac{k}{N} \wedge t\right)-h_{1}\left(\frac{k-1}{N} \wedge t\right)\right]-\left[h_{2}\left(\frac{k}{N} \wedge t\right)-h_{2}\left(\frac{k-1}{N} \wedge t\right)\right]\right\} \\
& +\int_{0}^{t}\left\{b\left(F_{N}^{(1)}(s), r_{1}(s)\right)-b\left(F_{N}^{(2)}(s), r_{2}(s)\right)\right\} \mathrm{d} s .
\end{aligned}
$$

For $0 \leqslant j \leqslant N-1$, set $I_{N, j}=[j / N,(j+1) / N]$; then, for $t \in I_{N, j}$, we have

$$
\left|\Psi_{N}(t)\right| \leqslant\left|\Psi_{N}\left(\frac{j}{N}\right)\right|+\sum_{i=1}^{3}\left|T_{i}(t)\right|
$$

where 


$$
\begin{aligned}
T_{1}(t)= & \left\{\sigma\left(F_{N}\left(h_{1}, r_{1}, u_{1}\right)\left(\frac{j}{N}\right), u_{1}\left(\frac{j}{N}\right)\right)-\sigma\left(F_{N}\left(h_{2}, r_{2}, u_{2}\right)\left(\frac{j}{N}\right), u_{2}\left(\frac{j}{N}\right)\right)\right\} \\
& \times\left\{h_{2}(t)-h_{2}\left(\frac{j}{N}\right)\right\}, \\
T_{2}(t)= & \sigma\left(F_{N}\left(h_{1}, r_{1}, u_{1}\right)\left(\frac{j}{N}\right), u_{1}\left(\frac{j}{N}\right)\right)\left\{\left\{h_{1}(t)-h_{1}\left(\frac{j}{N}\right)\right\}-\left\{h_{2}(t)-h_{2}\left(\frac{j}{N}\right)\right\}\right\}, \\
T_{3}(t)= & \int_{j / N}^{t}\left\{b\left(F_{N}\left(h_{1}, r_{1}, u_{1}\right)(s), r_{1}(s)\right)-b\left(F_{N}\left(h_{2}, r_{2}, u_{2}\right)(s), r_{2}(s)\right)\right\} \mathrm{d} s .
\end{aligned}
$$

The Lipschitz condition on $\sigma$ implies

$$
\begin{aligned}
T_{1}(t) \leqslant & C\left\{\left|F_{N}\left(h_{1}, r_{1}, u_{1}\right)\left(\frac{j}{N}\right)-F_{N}\left(h_{2}, r_{2}, u_{2}\right)\left(\frac{j}{N}\right)\right|+\left|u_{1}\left(\frac{j}{N}\right)-u_{2}\left(\frac{j}{N}\right)\right|\right\} \\
& \times\left|h_{2}(t)-h_{2}\left(\frac{j}{N}\right)\right| \\
\leqslant & C \cdot\left\{\left|\Psi_{N}\left(\frac{j}{N}\right)\right|+\left\|u_{1}-u_{2}\right\|_{\infty}\right\} \cdot 2\left\|h_{2}\right\|_{\infty} .
\end{aligned}
$$

Since $\sigma$ is bounded, we have

$$
\begin{aligned}
T_{2}(t) & \leqslant\|\sigma\|_{\infty} \cdot \sup _{t \in I_{N, j}}\left\{\left|h_{1}(t)-h_{2}(t)\right|+\left|h_{1}\left(\frac{j}{N}\right)-h_{2}\left(\frac{j}{N}\right)\right|\right\} \\
& \leqslant C \cdot\left\|h_{1}-h_{2}\right\|_{\infty} .
\end{aligned}
$$

The Lipschitz condition on $b$ implies

$$
T_{3}(t) \leqslant C\left\|r_{1}-r_{2}\right\|_{1 / L(1-\alpha)}+C \int_{j / N}^{t} \sup _{\frac{j}{N} \leqslant v \leqslant s}\left\{\left|F_{N}\left(h_{1}, r_{1}, u_{1}\right)_{v}-F_{N}\left(h_{2}, r_{2}, u_{2}\right)_{v}\right|\right\} \mathrm{d} s .
$$

Gronwall's lemma implies that, for $\left\|h_{1}\right\|_{\infty} \vee\left\|h_{2}\right\|_{\infty} \leqslant C$,

$$
\sup _{t \in I_{N, j}}\left|\Psi_{N}(t)\right| \leqslant C\left[\left|\Psi_{N}\left(\frac{j}{N}\right)\right|+\left\|h_{1}-h_{2}\right\|_{\infty}+\left\|r_{1}-r_{2}\right\|_{1 / L(1-\alpha)}+\left\|u_{1}-u_{2}\right\|_{\infty}\right] ;
$$

hence, for $t=(j+1) / N$,

$$
\left|\Psi_{N}\left(\frac{j+1}{N}\right)\right| \leqslant C\left[\left|\Psi_{N}\left(\frac{j}{N}\right)\right|+\left\|h_{1}-h_{2}\right\|_{\infty}+\left\|r_{1}-r_{2}\right\|_{1 / L(1-\alpha)}+\left\|u_{1}-u_{2}\right\|_{\infty}\right] .
$$

This, in turn, implies that, for $\left\|h_{1}\right\|_{\infty} \vee\left\|h_{2}\right\|_{\infty} \leqslant C$, there exists $C_{N}>0$ such that 


$$
\sup _{0 \leqslant j \leqslant N-1}\left|\Psi_{N}\left(\frac{j}{N}\right)\right| \leqslant C_{N}\left[\left\|h_{1}-h_{2}\right\|_{\infty}+\left\|r_{1}-r_{2}\right\|_{1 / L(1-\alpha)}+\left\|u_{1}-u_{2}\right\|_{\infty}\right] .
$$

Finally, (4.7) and (4.8) yield (4.6).

Hence, using (4.5), we see that to prove (4.4) it suffices to check that, for any $k \in\{1,2, \ldots, N\}$ there exists a constant $C>0$, such that

$$
\begin{aligned}
& \sup _{\frac{k-1}{N} \leqslant s<t \leqslant \frac{k}{N}} \frac{\left|\Psi_{N}(t)-\Psi_{N}(s)\right|}{|t-s|^{\alpha}} \\
& \quad \leqslant C\left(\left\|h_{1}-h_{2}\right\|_{\alpha}+\left\|r_{1}-r_{2}\right\|_{1 / L(1-\alpha)}+\left\|u_{1}-u_{2}\right\|_{\infty}+\sup _{t \in[0,1]}\left|\Psi_{N}(t)\right|\right) .
\end{aligned}
$$

Indeed, for $(k-1) / N \leqslant s<t \leqslant k / N$,

$$
F_{N}^{(i)}(t)-F_{N}^{(i)}(s)=\sigma\left(F_{N}^{(i)}\left(\frac{k-1}{N}\right), u_{i}\left(\frac{k-1}{N}\right)\right)\left[h_{i}(t)-h_{i}(s)\right]+\int_{s}^{t} b\left(F_{N}^{(i)}(v), r_{i}(v)\right) \mathrm{d} v .
$$

Then, using the Lipschitz conditions on $\sigma$ and $b$, the boundedness of $\sigma$ and Cauchy-Schwarz inequality, it is easy to see that

$$
\begin{aligned}
\left|\Psi_{N}(t)-\Psi_{N}(s)\right| & \mid \sigma\left(F_{N}^{(1)}\left(\frac{k-1}{N}\right), u_{1}\left(\frac{k-1}{N}\right)\right)\left[h_{1}(t)-h_{1}(s)\right] \\
& -\sigma\left(F_{N}^{(2)}\left(\frac{k-1}{N}\right), u_{2}\left(\frac{k-1}{N}\right)\right)\left[h_{2}(t)-h_{2}(s)\right] \mid \\
& +\left|\int_{s}^{t}\left[b\left(F_{N}^{(1)}(v), r_{1}(v)\right)-\int_{s}^{t} b\left(F_{N}^{(2)}(v), r_{2}(v)\right)\right] \mathrm{d} v\right| \\
\leqslant & \left|\sigma\left(F_{N}^{(1)}\left(\frac{k-1}{N}\right), u_{1}\left(\frac{k-1}{N}\right)\right)\left\{\left[h_{1}(t)-h_{1}(s)\right]-\left[h_{2}(t)-h_{2}(s)\right]\right\}\right| \\
& +\left|\left\{\sigma\left(F_{N}^{(1)}\left(\frac{k-1}{N}\right), u_{1}\left(\frac{k-1}{N}\right)\right)-\sigma\left(F_{N}^{(2)}\left(\frac{k-1}{N}\right), u_{2}\left(\frac{k-1}{N}\right)\right)\right\}\left[h_{2}(t)-h_{2}(s)\right]\right| \\
& +\left|\int_{s}^{t}\left\{\left[b\left(F_{N}^{(1)}(v), r_{1}(v)\right)-b\left(F_{N}^{1}(v), r_{2}(v)\right)\right]+\left[b\left(F_{N}^{(1)}(v), r_{2}(v)\right)-b\left(F_{N}^{(2)}(v), r_{2}(v)\right)\right]\right\} \mathrm{d} v\right| \\
\leqslant & C\left\{\left|\left(h_{1}(t)-h_{2}(t)\right)-\left(h_{1}(s)-h_{2}(s)\right)\right| .\right. \\
& +\left(\Psi_{N}\left(\frac{k-1}{N}\right)+\left|u_{1}\left(\frac{k-1}{N}\right)-u_{2}\left(\frac{k-1}{N}\right)\right|\right)\left|h_{2}(t)-h_{2}(s)\right| \\
& \left.+\int_{s}^{t}\left(\left|\Psi_{N}(v)\right|+\left|r_{1}(v)-r_{2}(v)\right|\right) \mathrm{d} v\right\} \\
\leqslant & C\left\{\left|\left(h_{1}(t)-h_{2}(t)\right)-\left(h_{1}(s)-h_{2}(s)\right)\right|+\left(\left\|\Psi_{N}\right\|\left\|_{\infty}+\right\| u_{1}-u_{2} \|_{\infty}\right)\left|h_{2}(t)-h_{2}(s)\right|\right. \\
& \left.+|t-s| \cdot\left\|\Psi_{N}\right\|_{\infty}+|t-s|^{\alpha}\left\|r_{1}-r_{2}\right\| L^{\{1 /(1-\alpha)\}}\right\} .
\end{aligned}
$$

Therefore, dividing by $|t-s|^{\alpha}$ and using the fact that $\left\|h_{2}\right\|_{\alpha}<C$, we obtain (4.9). 


\subsection{Uniform convergence of $F_{N}$ to $F$ on $\Gamma_{a} \times \operatorname{supp} Y \times \operatorname{supp} Z$}

To verify assertion (a)(ii) of Theorem 3.2, we first prove the following:

Lemma 4.2. For any $a>0$,

$$
\sup _{N} \sup _{\|h\|_{\mathscr{H}} \leqslant a(r, u) \in \operatorname{supp} Y \times \operatorname{supp} Z}\left(\left\|\mathrm{~F}_{N}(h, r, u)(\cdot)\right\|_{\infty} \vee\|F(h, r, u)(\cdot)\|_{\infty}\right)<\infty,
$$

and

$$
\lim _{N \rightarrow \infty} \sup _{\|h\|_{\mathscr{F}} \leqslant a} \sup _{(r, u) \in \operatorname{supp} Y \times \operatorname{supp} Z}\left\|F_{N}(h, r, u)(\cdot)-F(h, r, u)(\cdot)\right\|_{\alpha}=0 .
$$

Proof. The proof of (4.10) is a straightforward application of Gronwall's lemma. We will check (4.11); for $h \in\left\{\|h\|_{\mathscr{H}} \leqslant a\right\}, r \in \operatorname{supp} Y, u \in \operatorname{supp} Z$ and $t \in[0,1]$, we have

$$
F(h, r, u)(t)-F(h, r, u)\left(\underline{t}_{N}\right)=\int_{\underline{t}_{N}}^{t}\left[\sigma(F(h, r, u)(s), u(s)) \dot{h}_{s}+b(F(h, r, u)(s), r(s))\right] \mathrm{d} s .
$$

Hence hypotheses $\left(\mathrm{H}_{0}\right)-\left(\mathrm{H}_{2}\right)$ on coefficients together with the Cauchy-Schwarz inequality and (4.10) yield the existence of a constant $C>0$ (depending on $\|\sigma\|_{\infty}$ and $a$ ) such that

$$
\left\|F(h, r, u)(t)-F(h, r, u)\left(\underline{t}_{N}\right)\right\| \leqslant C\left(\frac{1}{\sqrt{N}}\|h\|_{\mathscr{H}}+\int_{\underline{t}_{N}}^{t}(1+|F(h, r, u)(s)|) \mathrm{d} s\right) \leqslant \frac{C}{\sqrt{N}} .
$$

Furthermore, for $t \in[0,1]$, using $\left(\mathrm{H}_{0}\right)-\left(\mathrm{H}_{2}\right)$ we have

$$
\begin{aligned}
&\left|F_{N}(h, r, u)(t)-F(h, r, u)(t)\right| \\
&=\mid \int_{0}^{t}\left\{\sigma(F(h, r, u)(s), u(s))-\sigma\left(F(h, r, u)\left(\underline{s}_{N}\right), u\left(\underline{s}_{N}\right)\right)\right\} \dot{h}(s) \mathrm{d} s \\
& \quad+\int_{0}^{t}\left\{\sigma\left(F(h, r, u)\left(\underline{s}_{N}\right), u\left(\underline{s}_{N}\right)\right)-\sigma\left(F_{N}(h, r, u)\left(\underline{s}_{N}\right), u\left(\underline{s}_{N}\right)\right)\right\} \dot{h}(s) \mathrm{d} s \\
& \quad+\int_{0}^{t}\left\{b(F(h, r, u)(s), r(s))-b\left(F_{N}(h, r, u)(s), r(s)\right)\right\} \mathrm{d} s \mid \\
& \leqslant C\left(\int_{0}^{t}\left\{\left|F(h, r, u)(s)-F(h, r, u)\left(\underline{s}_{N}\right)\right|+\left|u(s)-u\left(\underline{s}_{N}\right)\right|\right\}\left|\dot{h}_{s}\right| \mathrm{d} s\right. \\
&\left.+\int_{0}^{t}\left|F(h, r, u)\left(\underline{s}_{N}\right)-F_{N}(h, r, u)\left(\underline{s}_{N}\right)\right|\left|\dot{h}_{s}\right| \mathrm{d} s+\int_{0}^{t}\left|F_{N}(h, r, u)(s)-F(h, r, u)(s)\right| \mathrm{d} s\right) .
\end{aligned}
$$

By the Cauchy-Schwarz inequality and (4.12), there exists a constant $C(a)>0$ such that, for $\|h\|_{\mathscr{B}} \leqslant a$, 


$$
\begin{aligned}
\sup _{v \leqslant t}\left|F(h, r, u)(v)-F_{N}(h, r, u)(v)\right|^{2} \\
\quad \leqslant C(a)\left(1 / N+\sup _{s \in[0,1]}\left|u(s)-u\left(\underline{s}_{N}\right)\right|^{2}+\int_{0}^{t} \sup _{v \leqslant s}\left|F(h, r, u)(v)-F_{N}(h, r, u)(v)\right|^{2} \mathrm{~d} s\right) .
\end{aligned}
$$

Hence, Gronwall's lemma yields

$$
\sup _{v \leqslant t}\left|F(h, r, u)(v)-F_{N}(h, r, u)(v)\right|^{2} \leqslant C(a)\left(\frac{1}{N}+\sup _{s \in[0,1]}\left|u(s)-u\left(\underline{s}_{N}\right)\right|^{2}\right) \cdot \mathrm{e}^{C(a)} .
$$

Since supp $Z$ is a compact subset of $\mathscr{C}^{\alpha, 0}\left([0,1], \mathbb{R}^{l}\right)$, Ascoli's theorem implies

$$
\lim _{N} \sup _{s \in[0,1]}\left|u(s)-u\left(\underline{s}_{N}\right)\right|=0
$$

then (4.16) and (4.15) imply

$$
\lim _{N \rightarrow \infty} \sup _{\|h\|_{\mathscr{B}} \leqslant a} \sup _{(r, u) \in \operatorname{supp} Y \times \operatorname{supp} Z}\left\|F_{N}(h, r, u)(\cdot)-F(h, r, u)(\cdot)\right\|_{\infty}=0 .
$$

On the other hand, it is not difficult to see that, for $h \in\left\{\|h\|_{\mathscr{B}} \leqslant a\right\}, r \in \operatorname{supp} Y, u \in \operatorname{supp} Z$ and $s, t \in[0,1]$, there exists a constant $C(a)>0$ such that

$$
\begin{aligned}
& \frac{\left|\left[F_{N}(h, r, u)(t)-F(h, r, u)(t)\right]-\left[F_{N}(h, r, u)(s)-F(h, r, u)(s)\right]\right|}{|t-s|^{\alpha}} \\
& \leqslant C(a)\left(\frac{1}{\sqrt{N}}+\sup _{v \in[0,1]}\left|u(v)-u\left(\underline{v}_{N}\right)\right|\right)|t-s|^{1 / 2-\alpha} \\
& \quad+C(a)\left\|F_{N}(h, r, u)(\cdot)-F(h, r, u)(\cdot)\right\|_{\infty}\left(|t-s|^{1 / 2-\alpha}+|t-s|^{1-\alpha}\right),
\end{aligned}
$$

which, together with (4.16) and (4.17), concludes the proof of the lemma.

\subsection{Relative compactness}

The aim of this subsection is to prove condition $(b)$ of Theorem 3.2, which follows from the following:

Lemma 4.3. Let $\lambda$ be the good rate function defined by (1.3), $0<\alpha<\frac{1}{2}$, and $K$ be a relatively compact subset of $\mathscr{C}^{\alpha, 0}\left([0,1], \mathbb{R}^{k}\right)$. Then, for each $N \geqslant 1, a>0$, the sets $F_{N}(K \times \operatorname{supp} Y \times \operatorname{supp} Z), \quad F_{N}(\{\lambda \leqslant a\} \times \operatorname{supp} Y \times \operatorname{supp} Z)$ and $F(\{\lambda \leqslant a\} \times \operatorname{supp} Y \times$ supp $Z$ ) are relatively compact in $\mathscr{C}^{\alpha, 0}\left([0,1], \mathbb{R}^{d}\right)$.

Proof. Since $K$ is relatively compact, it is bounded in $\mathscr{C}^{a, 0}\left([0,1], \mathbb{R}^{d}\right)$. Fix $N \geqslant 1$, for $h \in K, r \in \operatorname{supp} Y$ and $u \in \operatorname{supp} Z$. Then by (4.2) and $\left(\mathrm{H}_{0}\right)-\left(\mathrm{H}_{2}\right)$ it is easy to see that there exists a constant $C_{N}>0$ such that 


$$
\begin{aligned}
\left\|F_{N}(h, r, u)(\cdot)\right\|_{\alpha} & \leqslant C_{N}\left(\|h\|_{\alpha}+\sup _{0 \leqslant s<t \leqslant 1}|t-s|^{(1-\alpha)}\right) \\
& \leqslant C_{N}\left(\|h\|_{\alpha}+1\right) .
\end{aligned}
$$

On the other hand, for $0<\delta<1 / N$ and $0<t-s<\delta$, there exists $1 \leqslant k \leqslant N$ such that either $(k-1) / N<s<k / N<t<(k+1) / N$ or $(k-1) / N \leqslant s<t<k / N$. The previous argument implies

$$
\begin{aligned}
\omega_{\alpha}\left(F_{N}(h, r, u), \delta\right) & =\sup _{0<|t-s|<\delta} \frac{\left|F_{N}(h, r, u)(t)-F_{N}(h, r, u)(s)\right|}{|t-s|^{\alpha}} \\
& \leqslant C_{N}\left(\omega_{\alpha}(h, \delta)+\delta^{(1-\alpha)}\right) .
\end{aligned}
$$

Since $h \in K$ is relatively compact in $\mathscr{C}^{\alpha, 0}\left([0,1], \mathbb{R}^{k}\right)$, then (4.18), (4.19) and Ascoli's result (Theorem 1.3) imply that $F_{N}(K \times \operatorname{supp} Y \times \operatorname{supp} Z)$ is relatively compact in $\mathscr{C}^{\alpha, 0}\left([0,1], \mathbb{R}^{d}\right)$. The same arguments prove the relative compactness of $F\left(\Gamma_{a} \times\right.$ $\operatorname{supp} Y \times \operatorname{supp} Z)$ in $\mathscr{C}^{\alpha, 0}\left([0,1], \mathbb{R}^{d}\right)$, since $\Gamma_{a}$ is a compact subset of $\mathscr{C}^{\alpha, 0}\left([0,1], \mathbb{R}^{k}\right)$.

\subsection{Large-deviation principle for $X_{N}^{\varepsilon}$ (as $\left.\varepsilon \rightarrow 0\right)$}

For $N \geqslant 1$, we prove that the family $X_{N}^{\varepsilon} \equiv F_{N}(\sqrt{\varepsilon} W, Y, Z)$ defined by (4.1) satisfies on $\mathscr{C}^{\alpha, 0}\left([0,1], \mathbb{R}^{d}\right)$ an LDP, and show that the rate function is of the form (3.1). Since $F_{N}$ is continuous on $\mathscr{C}^{\alpha, 0}\left([0,1], \mathbb{R}^{k}\right) \times L^{1 /(1-\alpha)}\left([0,1], \mathbb{R}^{m}\right) \times \mathscr{C}^{\alpha, 0}\left([0,1], \mathbb{R}^{l}\right)$, we use a version of the 'contraction principle'. Schilder's theorem implies that $\sqrt{\varepsilon} W$ satisfies an LDP on $\mathscr{C}^{\alpha, 0}\left([0,1], \mathbb{R}^{k}\right)$ with rate function $\lambda$ defined by (1.3).

For $N \geqslant 1$, define

$$
\lambda_{N}(f)=\inf \left\{\lambda(h), h \in \mathscr{B}\left([0,1], \mathbb{R}^{d}\right): \exists r \in \operatorname{supp} Y, \exists u \in \operatorname{supp} Z \text { s.t. } F_{N}(h, r, u) \equiv f\right\},
$$

and let $\lambda_{N}^{*}(f)$ be its 1sc regularization, i.e., $\lambda_{N}^{*}(f)=\lim _{a \rightarrow 0} \inf _{g \in B_{a}(f, a)} \lambda_{N}(g)$. An argument similar to that in the proof of Theorem 3.2 shows that $\lambda_{N}^{*}$ is a good rate function, and we check that $\left(X_{N}^{\varepsilon}\right)$ satisfies an LDP with rate function $\lambda_{N}^{*}$.

We first check the large-deviation lower bound.

Lemma 4.4. Let $G$ be an open subset of $\mathscr{C}^{\alpha, 0}\left([0,1], \mathbb{R}^{d}\right)$; then

$$
\liminf _{\varepsilon \rightarrow 0} \varepsilon \ln \mathbb{P}\left\{X_{N}^{\varepsilon} \in G\right\} \geqslant-\inf \left\{\lambda_{N}^{*}(f) ; f \in G\right\} .
$$

Proof. Assume $G \neq \varnothing$, and let $f \in G$ be such that $\lambda_{N}^{*}(f)<\infty$; we prove

$$
\liminf _{\varepsilon \rightarrow 0} \varepsilon \ln \mathbb{P}\left\{X_{N}^{\varepsilon} \in G\right\} \geqslant-\lambda_{N}^{*}(f) .
$$

By definition, given $\delta, \eta>0$, there exist $(h, r, u) \in \mathscr{H}\left([0,1], \mathbb{R}^{d}\right) \times \operatorname{supp} Y \times \operatorname{supp} Z$ such that

$$
\left\|F_{N}(h, r, u)(\cdot)-f(\cdot)\right\|_{\alpha}<\eta \quad \text { and } \quad \lambda(h) \leqslant \lambda_{N}^{*}(f)+\delta .
$$


We can choose $\eta$ small enough to ensure $B_{\alpha}(f, 2 \eta) \subset G$. The continuity of $F_{N}(\cdot, r, u)$ on $\mathscr{C}^{\alpha, 0}\left([0,1], \mathbb{R}^{k}\right) \times L^{1 /(1-\alpha)}\left([0,1], \mathbb{R}^{m}\right) \times \mathscr{C}^{\alpha, 0}\left([0,1], \mathbb{R}^{l}\right)$ implies the existence of $\beta>0$ such that $\|Y-r\|_{1 / L(1-\alpha)}<\beta / L,\|Z-u\|_{\alpha}<\beta,\|\sqrt{\varepsilon} W-h\|_{\alpha}<\beta$ and

$$
\left\{\|\sqrt{\varepsilon} W-h\|_{\alpha}<\beta\right\} \cap\left\{\|\mathrm{Y}-\mathrm{r}\|_{1 / L(1-\alpha)}<\beta\right\} \cap\left\{\|\mathrm{Z}-u\|_{\alpha}<\beta\right\} \subset\left\{X_{N}^{\varepsilon} \in G\right\} .
$$

Since $(r, u) \in \operatorname{supp} Y \times \operatorname{supp} Z, P\left(\|Y-r\|_{1 / L(1-\alpha)}<\beta,\|Z-u\|_{\infty}<\beta\right)>0$ and the independence of $W$ and $(Y, Z)$ yield

$$
\begin{aligned}
\liminf _{\varepsilon \rightarrow 0} \varepsilon \ln P\left\{X_{N}^{\varepsilon} \in G\right\} & \geqslant \liminf _{\varepsilon \rightarrow 0} \varepsilon \ln P\left\{\|\sqrt{\varepsilon} W-h\|_{\alpha}<\beta\right\} \\
& \geqslant-\lambda(h) \geqslant-\lambda_{N}^{*}(f)-\delta .
\end{aligned}
$$

Letting $\delta \rightarrow 0$, we conclude the proof.

We now prove the large-deviation upper bound.

Lemma 4.5. Let $A$ be a closed subset of $\mathscr{C}^{\alpha, 0}\left([0,1], \mathbb{R}^{d}\right)$; then

$$
\limsup _{\varepsilon \rightarrow 0} \varepsilon \ln P\left\{X_{N}^{\varepsilon} \in A\right\} \leqslant-\inf \left\{\lambda_{N}^{*}(f) ; f \in A\right\} .
$$

The proof of Lemma 4.5 is obvious if $Y$ has compact support; in general, this depends on some technical results.

Lemma 4.6. Let $A$ be a closed subset of $\mathscr{C}^{\alpha, 0}\left([0,1], \mathbb{R}^{d}\right)$; then

$$
\limsup _{\varepsilon \rightarrow 0} \varepsilon \ln P\left\{X_{N}^{\varepsilon} \in A\right\} \leqslant-\inf \{\lambda(h) ; h \in \overline{\mathscr{K}(A)}\},
$$

where $\overline{\mathscr{K}(A)}$ is the closure in $\mathscr{C}^{\alpha, 0}\left([0,1], \mathbb{R}^{d}\right)$ of

$$
\mathscr{K}(A)=\left\{h \in \mathscr{C}^{\alpha, 0}\left([0,1], \mathbb{R}^{d}\right): \exists r \in \operatorname{supp} Y, \exists u \in \operatorname{supp} Z \text { s.t. } F_{N}(h, r, u) \in A\right\} .
$$

Proof. The proof follows immediately from Schilder's theorem and the inclusions

$$
\left\{X_{N}^{\varepsilon} \in A\right\}=\left\{F_{N}(\sqrt{\varepsilon} W, Y, Z) \in A\right\} \subset\{\sqrt{\varepsilon} W \in \mathscr{K}(A)\} .
$$

Proof of Lemma 4.5. Let $A$ be a closed subset $A$ of $\mathscr{C}^{\alpha, 0}\left([0,1], \mathbb{R}^{d}\right)$; by Lemma 4.6, it suffices to check that

$$
\inf \left\{\lambda_{N}^{*}(f) ; f \in A\right\} \leqslant \inf \{\lambda(h) ; h \in \overline{\mathscr{K}(A)}\} .
$$

Let $h \in \overline{\mathscr{K}}(A)$; then there exist sequences $h_{n} \in \mathscr{K}(A), r_{n} \in \operatorname{supp} Y$ and $u_{n} \in \operatorname{supp} Z$ such that $g_{n}=F_{N}\left(h_{n}, r_{n}, u_{n}\right) \in A$ and $h_{n}$ converges to $h$ in $\mathscr{C}^{\alpha, 0}\left([0,1], \mathbb{R}^{d}\right)$. Since $h_{n}$ is relatively compact in $\mathscr{C}^{\alpha, 0}\left([0,1], \mathbb{R}^{k}\right)$ it follows from Lemma 4.3 that $g_{n}$ is also relatively compact in $\mathscr{C}^{\alpha, 0}\left([0,1], \mathbb{R}^{d}\right)$; thus (by extracting a subsequence) we may and do assume that $g_{n}$ converges in $\mathscr{C}^{a, 0}\left([0,1], \mathbb{R}^{d}\right)$, say to $g$. Note that $g_{n} \in A$ and $A$ is closed, so that $g \in A$. 
Set $\tilde{g}_{n}=F_{N}\left(h, r_{n}, u_{n}\right)$; (4.4) implies that $\lim _{n}\left\|g_{n}-\tilde{g}_{n}\right\|_{\alpha}=0$. Finally, for each $h \in \overline{\mathscr{K}(A)}$, by definition of $\lambda_{N}^{*}$ :

$$
\begin{aligned}
\inf \left\{\lambda_{N}^{*}(f) ; f \in A\right\} & \leqslant \lambda_{N}^{*}(g) \\
& \leqslant \liminf _{n \rightarrow \infty} \lambda_{N}\left(\tilde{g}_{n}\right) \\
& \leqslant \lambda(h),
\end{aligned}
$$

and (4.21) is proved.

\subsection{Exponential approximations}

We finally show that $\left\{X_{N}^{\varepsilon}\right\}$ defined by (4.1) are exponentially good approximations of $\left\{X^{\varepsilon}\right\}$ defined by (2.1). Let us at first establish the following approximation.

Lemma 4.7. For any $\delta>0$,

$$
\limsup _{N \rightarrow \infty} \limsup _{\varepsilon \rightarrow 0} \varepsilon \ln P\left(\sup _{t \in[0,1]}\left|X_{N}^{\varepsilon}(t)-X^{\varepsilon}(t)\right|>\frac{\delta}{N^{\alpha}}\right)=-\infty .
$$

Proof. Since the drift coefficient $b$ is not neccessarily bounded, to prove (4.22), let us introduce some auxilliary results. Let $0<\alpha<\beta<\frac{1}{2}$ and $0<\gamma<\beta-\alpha$; then, by Theorem 1.4 ,

$$
\begin{aligned}
\limsup _{\varepsilon \rightarrow 0} \varepsilon \ln P\left(\sup _{1 \leqslant k \leqslant N} \mid \sqrt{\varepsilon}\left(W_{k / N}-W_{(k-1) / N} \mid \geqslant N^{\gamma-\beta}\right)\right. & \leqslant \limsup _{\varepsilon \rightarrow 0} \varepsilon \ln P\left(\|\sqrt{\varepsilon} W\|_{\beta} \geqslant N^{\gamma}\right) \\
& \leqslant-\inf \left\{\frac{1}{2}\|h\|_{\mathscr{H}}^{2} ;\|h\|_{\beta} \geqslant N^{\gamma}\right\} \\
& \leqslant-\frac{1}{2} N^{2 \gamma} .
\end{aligned}
$$

Indeed, if $h \in \mathscr{H}\left([0,1], \mathbb{R}^{d}\right)$ satisfies $\|h\|_{\beta} \geqslant N^{\gamma}$, the Cauchy-Schwarz inequality implies $\|h\|_{\mathscr{H}} \geqslant N^{\gamma}$.

Define the set

$$
B_{\beta, \gamma, \varepsilon}=\left\{\sup _{1 \leqslant k \leqslant N} \mid \sqrt{\varepsilon}\left(W_{k / N}-W_{(k-1) / N} \mid \leqslant N^{\gamma-\beta}\right\} \cap\left\{\|\sqrt{\varepsilon} W\|_{\beta} \leqslant N^{\gamma}\right\} ;\right.
$$

by (4.23),

$$
\lim _{N \rightarrow \infty} \limsup _{\varepsilon \rightarrow 0} \varepsilon \ln P\left(B_{\beta, \gamma, \varepsilon}^{c}\right)=-\infty .
$$

Furthermore, on the set $B_{\beta, \gamma, \varepsilon}$, by Gronwall's lemma and the assumptions of the coefficients $\sigma, b$ (Section 2), for $t \in[0,1]$, we deduce the existence of a constant $C>0$ such that 


$$
\begin{aligned}
\left|X_{N}^{\varepsilon}(t)\right| & \leqslant C\left\{\sum_{k=1}^{N} \sqrt{\varepsilon}\left|W_{k / N \wedge t}-W_{(k-1) / N \wedge t}\right|+\int_{0}^{t}\left(1+\left|X_{N}^{\varepsilon}(s)\right|\right) \mathrm{d} s\right\} \\
& \leqslant C\left\{N^{\gamma+1-\beta}+\int_{0}^{t}\left|X_{N}^{\varepsilon}(s)\right| \mathrm{d} s\right\} \\
& \leqslant C N^{\gamma+1-\beta} .
\end{aligned}
$$

To prove (4.22), set $\Psi_{N}^{\varepsilon}(\cdot)=X_{N}^{\varepsilon}(\cdot)-X^{\varepsilon}(\cdot)$ and $\underline{t}_{N}=[N t] / N$; then for $t \in[0,1], \Psi_{N}^{\varepsilon}(t)$ satisfies

$$
\begin{aligned}
\Psi_{N}^{\varepsilon}(t)= & \sqrt{\varepsilon} \int_{0}^{t}\left\{\sigma\left(X_{N}^{\varepsilon}\left(\underline{s}_{N}\right), Z\left(\underline{s}_{N}\right)\right)-\sigma\left(X^{\varepsilon}(s), Z(s)\right)\right\} \mathrm{d} W_{s} \\
& +\int_{0}^{t}\left\{b\left(X_{N}^{\varepsilon}(s), Y(s)\right)-b\left(X^{\varepsilon}(s), Y(s)\right)\right\} \mathrm{d} s .
\end{aligned}
$$

For $\rho>0$, we define

$$
\begin{aligned}
& \tau_{N, \varepsilon}^{\rho}(\omega)=\inf \left\{t \geqslant 0 ;\left|X_{N}^{\varepsilon}(t, \omega)-X_{N}^{\varepsilon}\left(\underline{t}_{N}, \omega\right)\right| \geqslant \frac{\rho}{N^{\alpha}}\right\} \wedge 1 \\
& \theta_{N, \varepsilon}^{\rho, \delta}(\omega)=\inf \left\{t \geqslant 0 ;\left|\Psi_{N}^{\varepsilon}(t, \omega)\right|>\frac{\delta}{N^{\alpha}}\right\} \wedge \tau_{N, \varepsilon}^{\rho}(\omega)
\end{aligned}
$$

and

$$
v_{N, \varepsilon}^{\rho}(t)=\int_{\Omega}\left(\frac{\rho^{2}}{N^{2 \alpha}}+\left|\Psi_{N}^{\varepsilon}\left(t \wedge \theta_{N, \varepsilon}^{\rho, \delta}(\omega), \omega\right)\right|^{2}\right)^{1 / \varepsilon} \mathrm{d} P
$$

Then clearly

$$
P\left(\sup _{t \in[0,1]}\left|X_{N}^{\varepsilon}(t)-X^{\varepsilon}(t)\right|>\frac{\delta}{N^{\alpha}}\right) \leqslant P\left(\tau_{N, \varepsilon}^{\rho}<1\right)+P\left(\theta_{N, \varepsilon}^{\rho, \delta}<1\right) .
$$

First, we apply Stroock's inequality (1.4) and expression (4.26), together with hypotheses $\left(\mathrm{H}_{0}\right)-\left(\mathrm{H}_{2}\right)$, to obtain the existence of a constant $\mathrm{C}>0$ such that 


$$
\begin{aligned}
P\left(\tau_{N, \varepsilon}^{\rho}<1\right) & \leqslant P\left(\tau_{N, \varepsilon}^{\rho}<1, B_{\beta, \gamma, \varepsilon}\right)+P\left(\tau_{N, \varepsilon}^{\rho}<1, B_{\beta, \gamma, \varepsilon}^{c}\right) \\
& \leqslant \sum_{k=1}^{N} P\left(\sup _{\frac{k-1}{N} \leqslant t \leqslant \frac{k}{N}}\left|X_{N}^{\varepsilon}(t)-X_{N}^{\varepsilon}\left(\frac{k-1}{N}\right)\right| \geqslant \frac{\rho}{N^{\alpha}}, B_{\beta, \gamma, \varepsilon}\right)+P\left(B_{\beta, \gamma, \varepsilon}^{c}\right) \\
& \leqslant C d N \exp \left\{-\frac{\left(\rho-C d N^{\gamma+1-\beta}\left(\frac{1}{N}\right)^{1-\alpha}\right)^{2}}{C \varepsilon d^{2}\left(\frac{1}{N}\right)^{1-2 \alpha}}\right\}+P\left(B_{\beta, \gamma, \varepsilon}^{c}\right) \\
& \leqslant C d N \exp \left\{-C N^{1-2 \alpha} / \varepsilon\right\}+P\left(B_{\beta, \gamma, \varepsilon}^{c}\right),
\end{aligned}
$$

since $N^{\gamma+\alpha-\beta} \rightarrow 0$ as $N \rightarrow \infty(\gamma<\beta-\alpha)$. Thus, using (4.25), we deduce

$$
\lim _{N} \limsup _{\varepsilon} \varepsilon \ln P\left(\tau_{N, \varepsilon}^{\rho}<1\right)=-\infty
$$

Since $\operatorname{supp} Z$ is a compact subset of $\mathscr{C}^{\alpha, 0}\left([0,1], \mathbb{R}^{l}\right)$, for every $\rho>0$ there exists $N_{0} \geqslant 1$ such that, for $N \geqslant N_{0}$,

$$
\sup _{0 \leqslant t \leqslant 1}\left|Z(t)-Z\left(\underline{t}_{N}\right)\right| \leqslant \rho N^{-\alpha}
$$

For $0<\varepsilon<\frac{1}{2}$, set $\rho_{N}=\rho / N^{\alpha}$ and $f_{\varepsilon, \rho}(y)=\left(\rho_{N}^{2}+|y|^{2}\right)^{1 / \varepsilon}$. Then an application of Itô's formula to $f_{\varepsilon, \rho}\left(\Psi_{N}^{\varepsilon}(t)\right)$ yields that

$$
f_{\varepsilon, \rho}\left(\Psi_{N}^{\varepsilon}\left(t \wedge \theta_{N, \varepsilon}^{\rho, \delta}\right)\right)-\int_{0}^{t \wedge \theta_{N, \varepsilon}^{\rho, \delta}} g_{\varepsilon, N}^{\rho}(s) \mathrm{d} s-\rho_{N}^{2 / \varepsilon},
$$

is a martingale, where, if $\langle\cdot, \cdot\rangle$ is the inner product in $\mathbb{R}^{d}$,

$$
\begin{aligned}
g_{\varepsilon, N}^{\rho}(t)= & \frac{2}{\varepsilon}\left(\rho_{N}^{2}+\left|\Psi_{N}^{\varepsilon}(t)\right|^{2}\right)^{1 / \varepsilon-1} \cdot\left\langle\Psi_{N}^{\varepsilon}(t),\left(b\left(X_{N}^{\varepsilon}(t), Y(t)\right)-b\left(X^{\varepsilon}(t), Y(t)\right)\right)\right\rangle \\
& +\frac{2}{\varepsilon}\left(\frac{1}{\varepsilon}-1\right) \varepsilon\left\|\left(\sigma\left(X_{N}^{\varepsilon}\left(\underline{t}_{N}\right), Z\left(\underline{t}_{N}\right)\right)-\sigma\left(X^{\varepsilon}(t), Z(t)\right)\right)^{*} \Psi_{N}^{\varepsilon}(t)\right\|^{2}\left(\rho_{N}^{2}+\left|\Psi_{N}^{\varepsilon}(t)\right|^{2}\right)^{1 / \varepsilon-2} \\
& +\left\|\sigma\left(X_{N}^{\varepsilon}\left(\underline{t}_{N}\right), Z\left(\underline{t}_{N}\right)\right)-\sigma\left(X^{\varepsilon}(t), Z(t)\right)\right\|^{2}\left(\rho_{N}^{2}+\left|\Psi_{N}^{\varepsilon}(t)\right|^{2}\right)^{1 / \varepsilon-1}
\end{aligned}
$$

For $0 \leqslant t \leqslant \tau_{N, \varepsilon}^{\rho}$, using (4.30), we have, for $N \geqslant N_{0}$ and $0<\varepsilon<\frac{1}{2}$, that there exists $C>0$ such that 


$$
\begin{aligned}
\left|g_{\varepsilon, N}^{\rho}(t)\right| \leqslant & C \frac{2}{\varepsilon}\left(\rho_{N}^{2}+\left|\Psi_{N}^{\varepsilon}(t)\right|^{2}\right)^{1 / \varepsilon} \frac{\left|\Psi_{N}^{\varepsilon}(t)\right|}{\rho_{N}^{2}+\left|\Psi_{N}^{\varepsilon}(t)\right|^{2}}\left|X_{N}^{\varepsilon}(t)-X^{\varepsilon}(t)\right| \\
& +C\left|\frac{1}{\varepsilon}-1\right|\left\{\rho_{N}^{2}+\left|Z\left(\underline{t}_{N}\right)-Z(t)\right|^{2}\right\} \frac{\left|\Psi_{N}^{\varepsilon}(t)\right|^{2}}{\rho_{N}^{2}+\left|\Psi_{N}^{\varepsilon}(t)\right|^{2}}\left(\rho_{N}^{2}+\left|\Psi_{N}^{\varepsilon}(t)\right|^{2}\right)^{1 / \varepsilon-1} \\
& \left.\left.+C\left\{\rho_{N}^{2}+\mid Z\right) \underline{t}_{N}\right)-\left.Z(t)\right|^{2}\right\}\left(\rho_{N}^{2}+\left|\Psi_{N}^{\varepsilon}(t)\right|^{2}\right)^{1 / \varepsilon-1} \\
\leqslant & C \frac{1}{\varepsilon} f_{\varepsilon, \rho}\left(\Psi_{N}^{\varepsilon}(t)\right) \frac{\left|\Psi_{N}^{\varepsilon}(t)\right| \rho_{N}}{\rho_{N}^{2}+C\left|\Psi_{N}^{\varepsilon}(t)\right|^{2}} \\
& +\left|\frac{1}{\varepsilon}-1\right| \frac{\rho_{N}^{2}}{\rho_{N}^{2}+\left|\Psi_{N}^{\varepsilon}(t)\right|^{2}} \frac{\left|\Psi_{N}^{\varepsilon}(t)\right|^{2}}{\rho_{N}^{2}+\left|\Psi_{N}^{\varepsilon}(t)\right|^{2}} f_{\varepsilon, \rho}\left(\Psi_{N}^{\varepsilon}(t)\right) \\
& +C\left|\frac{1}{\varepsilon}-1\right| \frac{\left|Z\left(\underline{t}_{N}\right)-Z(t)\right|^{2}}{\rho_{N}^{2}+\left|\Psi_{N}^{\varepsilon}(t)\right|^{2}} \frac{\left|\Psi_{N}^{\varepsilon}(t)\right|^{2}}{\rho_{N}^{2}+\left|\Psi_{N}^{\varepsilon}(t)\right|^{2}} f_{\varepsilon, \rho}\left(\Psi_{N}^{\varepsilon}(t)\right) \\
& +C \frac{\rho_{N}^{2}+\left|Z\left(\underline{t}_{N}\right)-Z(t)\right|^{2}}{\rho_{N}^{2}+\left|\Psi_{N}^{\varepsilon}(t)\right|^{2}} f_{\varepsilon, \rho}\left(\Psi_{N}^{\varepsilon}(t)\right) \\
\leqslant & C\left\{\left(\frac{1}{\varepsilon}+1\right)+\left(1+\frac{\left|Z\left(\underline{t}_{N}\right)-Z(t)\right|^{2}}{\rho_{N}^{2}+\left|\Psi_{N}^{\varepsilon}(t)\right|^{2}}\right)\right\} f_{\varepsilon, \rho}\left(\Psi_{N}^{\varepsilon}(t)\right) \\
\leqslant & \frac{C}{\varepsilon} f_{\varepsilon, \rho}\left(\Psi_{N}^{\varepsilon}(t)\right) .
\end{aligned}
$$

This, together with Doob's stopping theorem, shows that there exists a constant $\tilde{C}<\infty$, independent of $N, \varepsilon, \rho$ and $N_{0}$, such that, for $N \geqslant N_{0}$,

$$
v_{N, \varepsilon}^{\rho}(t) \leqslant \rho_{N}^{2 / \varepsilon}+\frac{\tilde{C}}{\varepsilon} \int_{0}^{t} v_{N, \varepsilon}^{\rho}(s) \mathrm{d} s, \quad t \in[0,1]
$$

(see, for example, Deuschel and Stroock 1989, p. 30). Therefore, for $N \geqslant N_{0}$,

$$
v_{N, \varepsilon}^{\rho}(1) \leqslant \exp \left\{\frac{1}{\varepsilon}(\tilde{C}+2 \ln \rho-2 \alpha \ln N)\right\}
$$

Since, for all $N \geqslant 1$,

$$
P\left(\theta_{N, \varepsilon}^{\rho, \delta}<1\right) \leqslant\left(\frac{\rho^{2}+\delta^{2}}{N^{2 \alpha}}\right)^{-1 / \varepsilon} v_{N, \varepsilon}^{\rho}(1)
$$

we conclude

$$
\lim _{\rho \rightarrow 0} \sup _{N} \limsup _{\varepsilon \rightarrow 0} \varepsilon \ln P\left(\theta_{N, \varepsilon}^{\rho, \delta}<1\right)=-\infty .
$$

This, together with (4.28) and (4.29), implies (4.22). 
Lemma 4.8. For any $\delta>0$,

$$
\limsup _{N \rightarrow \infty} \limsup _{\varepsilon \rightarrow 0} \varepsilon \ln P\left(\left\|X_{N}^{\varepsilon}(t)-X^{\varepsilon}(t)\right\|_{\alpha}>\delta\right)=-\infty .
$$

Proof. Applying (4.5) to $\Psi_{N}^{\varepsilon}(\cdot)=X_{N}^{\varepsilon}(\cdot)-X^{\varepsilon}(\cdot)$ and Lemma 4.7, we see that it suffices to prove

$$
\limsup _{N \rightarrow \infty} \limsup _{\varepsilon \rightarrow 0} \varepsilon \ln P\left(\max _{1 \leqslant k \leqslant N}\left\{\sup _{\frac{k-1}{N} \leqslant s<t \leqslant \frac{k}{N}} \frac{\left|\Psi_{N}(t)-\Psi_{N}(s)\right|}{|t-s|^{\alpha}}\right\}>\delta\right)=-\infty .
$$

Thus, by Lemma 1.5 and an argument similar to that in the proof of the previous lemmas, it is easy to see that, for $0<\alpha<\beta<\frac{1}{2}$ and $0<\gamma<\beta-\alpha$,

$$
\limsup _{N \rightarrow \infty} \limsup _{\varepsilon \rightarrow 0} \varepsilon \ln P\left(\max _{1 \leqslant k \leqslant N}\left\{\sup _{\frac{k-1}{N} \leqslant s<t \leqslant \frac{k}{N}} \frac{\left|\Psi_{N}(t)-\Psi_{N}(s)\right|}{|t-s|^{\alpha}}\right\}>\delta, B_{\beta, \gamma, \varepsilon}\right)=-\infty,
$$

where $B_{\beta, \gamma, \varepsilon}$ is defined by (4.24); thus (4.25) concludes the proof.

\section{Acknowledgements}

The author would like to thank Professor Annie Millet for having read this paper and having made a number of very useful suggestions. Thanks are also due to the referees for their comments.

\section{References}

Azencott, R. (1980) Grandes déviations et applications. In P.L. Hennequin (ed.), École d'Été de Probabilités de Saint-Flour VIII, Lecture Notes in Math. 774, pp. 1-176. Berlin: Springer-Verlag.

Baldi, P., Ben Arous, G. and Kerkyacharian, G. (1992) Large deviation and Strassen law in Hölder norm. Stochastic Process. Appl., 42, 171-180.

Ben Arous, G. and Ledoux, M. (1994) Grandes déviations de Freidlin-Wentzell en norme Höldérienne. In J. Azéma, P.-A. Meyer and M. Yor (eds), Séminaire de Probabilités XXVIII, Lecture Notes in Math. 1583, pp. 293-299. Berlin: Springer-Verlag.

Bezuidenhout, C. (1987) A large deviations principle for small perturbations of random evolution equations. Ann. Probab., 15, 646-658.

Chernoff, H. (1952) A measure of asymptotic efficiency for tests of hypothesis based on the sum of observations. Ann. Math. Statist., 23, 493-507.

Dawson, D. and Gärtner, J. (1987) Large deviations from the McKean-Vlasov limit for weakly interacting diffusions. Stochastics, 20, 247-308.

Dembo, A. and Zeitouni, O. (1993) Large Deviations Techniques and Applications. Boston: Jones and Bartlett.

Deuschel, J.D. and Stroock, D.W. (1989) Large Deviations. New York: Academic Press. 
Freidlin, M.I. and Wentzell, A. (1984) Random Perturbations of Dynamical Systems. Berlin: SpringerVerlag.

Gihman, I.I. and Skorohod, A.V. (1972) Stochastic Differential Equations. Berlin: Springer-Verlag.

Griego, R. and Hersh, J. (1971) Random evolutions, Markov chains and systems of partial differential equations. Trans. Amer. Math. Soc., 156, 405-418.

Heath, D. (1969) Probabilistic analysis of hyperbolic systems of partial differential equations. Ph.D. thesis, University of Illinois.

Hu, Y.-J. (1997) A large deviation principle for small perturbations of random evolution equations in Hölder norm, Stochastic Process. Appl., 68, 83-99.

Pérez-Abreu, V. and Tudor, C. (1994) Large deviations for a class of chaos expansions. J. Theoret. Probab., 7, 757-765.

Ruelle, D. (1969) Statistical Mechanics. Rigorous Results. New York: Benjamin.

Schilder, M. (1966) Some asymptotic formulas for Wiener integrals. Trans. Amer. Math. Soc., 125, 63-85.

Stroock, D.W. (1981) Some applications of stochastic calculus to partial differential equations, In P.L. Hennequin (ed.), École d'été de probabilités de Saint Flour XI, Lecture Notes in Math. 976, pp. 267-382. Berlin: Springer-Verlag.

Varadhan, S.R.S. (1967) Diffusion processes in a small time interval. Comm. Pure Appl. Math., 20, $659-685$.

Varadhan, S.R.S. (1984) Large Deviations and Applications. Philadelphia: Society of Industrial and Applied Mathematics.

Received March 1999 and revised October 1999 